\title{
A METHOD OF TREATING BOUNDARY \\ SINGULARITIES IN TIME-DEPENDENT \\ PROBLEMS
}

by

GRAHAM E. BELL

Department of Mathematics, Brunel University, Uxbridge. 
A Method of Treating Boundary Singularities

in Time-Dependent Problems

\author{
GRAHAM E.. BELL* \\ Department of Mathematics, Brunel University, Uxbridge
}

\begin{abstract}
A method is presented for treating singularities which occur in solutions of Parabolic partial differential equations due to sharp corners in the boundary. The method is essentially an extension of the method due to Motz (1946) for solving Elliptic problems and approximates to the analytical form of the singularity in terms of neighbouring function values at each time step. It is used in conjunction with the simple explicit finite-difference scheme and subsequently the overall method is explicit.
\end{abstract}

This work was sponsored by a Science Research Council (CAPS) Award in conjunction with the Gas Council. 


\section{Introduction.}

In recent years much attention has been given to elliptic partial differential equations in domains which contain re-entrant corners but little work has been done on corresponding parabolic problems. Standard finite difference and, to a lesser extent, finite element methods fail to produce accurate approximations to the exact solution in the neighbourhood of a singularity.

In the following method the finite difference approximation in such a neighbourhood is replaced by a truncated series representation of the exact solution at points close to the corner. The coefficients of this truncated series are estimated at each time step in terms of the solution values at points where the influence of the singularity is neglected and which have been derived by an explicit finite difference process from the previous time step. It is also possible to implement the method with an implicit scheme but it is computationally desirable to avoid the solution of awkward systems of equations at each time increment. The analytical form of the exact solution around a general sharp corner for the transient heat flow or diffusion equation is derived in section 2 and is applied in section 3 with reference to the re-entrant corner. The number of terms included in the truncated form of the solution is optional and is discussed later in terms of two model problems. 
2. Singularities in Time-Dependent Problems.

Consider the two dimensional, time-dependent, diffusion equation in a bounded domain containing boundary singularities due to the presence of sharp corners in the boundary where

$$
\begin{array}{rlrl}
\mathrm{v}^{2} \mathrm{u} & =\frac{\partial \mathrm{u}}{\partial \mathrm{t}} & (\mathrm{x}, \mathrm{y}) \in \mathrm{R}, \quad \mathrm{t}>0, \\
\mathrm{u}=\mathrm{f}(\mathrm{x}, \mathrm{y}) & (\mathrm{x}, \mathrm{y}) \quad \in \partial \mathrm{R}, \quad \mathrm{t} \geq 0, \\
\frac{\partial \mathrm{u}}{\partial \mathrm{v}}=0 & (\mathrm{x}, \mathrm{y}) \quad \in \partial \mathrm{R}_{1}, \quad \mathrm{t} \geq 0 \\
\mathrm{a} \frac{\partial \mathrm{u}}{\partial \mathrm{v}}+\mathrm{b}(\mathrm{u}-\mathrm{v}) & =0, & (\mathrm{x}, \mathrm{y}) \quad \in \mathrm{\partial R}_{2}, \quad \mathrm{t} \geq 0
\end{array}
$$

and $\mathrm{a}, \mathrm{b} v$ are constants.

The region $\mathrm{R}$ is a bounded domain with boundary $\partial \mathrm{R}=\partial \mathrm{R}_{1} \cup \partial \mathrm{R}_{2}$, where $\partial \mathrm{R}_{1}$ is that part of the boundary which contains the singularities.

Taking a typical sharp corner $\mathrm{P}$, we can express (2.1) in polar coordinates $\mathrm{r}, \theta$ centred at P, (Fig. 1), in the form

$$
\frac{\partial^{2} \mathrm{u}}{\partial \mathrm{r}^{2}}+\frac{1}{\mathrm{r}} \frac{\partial \mathrm{u}}{\partial \mathrm{r}}+\frac{1}{\mathrm{r}^{2}} \frac{\partial^{2} \mathrm{u}}{\partial \theta^{2}}=\frac{\partial \mathrm{u}}{\partial \mathrm{t}}
$$

The boundary conditions (2.3) in the neighbourhood of $\mathrm{P}$ become

$$
\frac{\partial \mathrm{u}}{\partial \theta}=0 \quad \text { at } \quad \theta=0, \theta_{0} \text {. }
$$

Letting the solution of (2.5) have the form

$$
\mathrm{u}=\mathrm{e}^{-\alpha^{2} \mathrm{t}} \mathrm{R}(\mathrm{r}) \psi(\theta)+\mathrm{w}(\mathrm{r}, \theta)
$$


4.

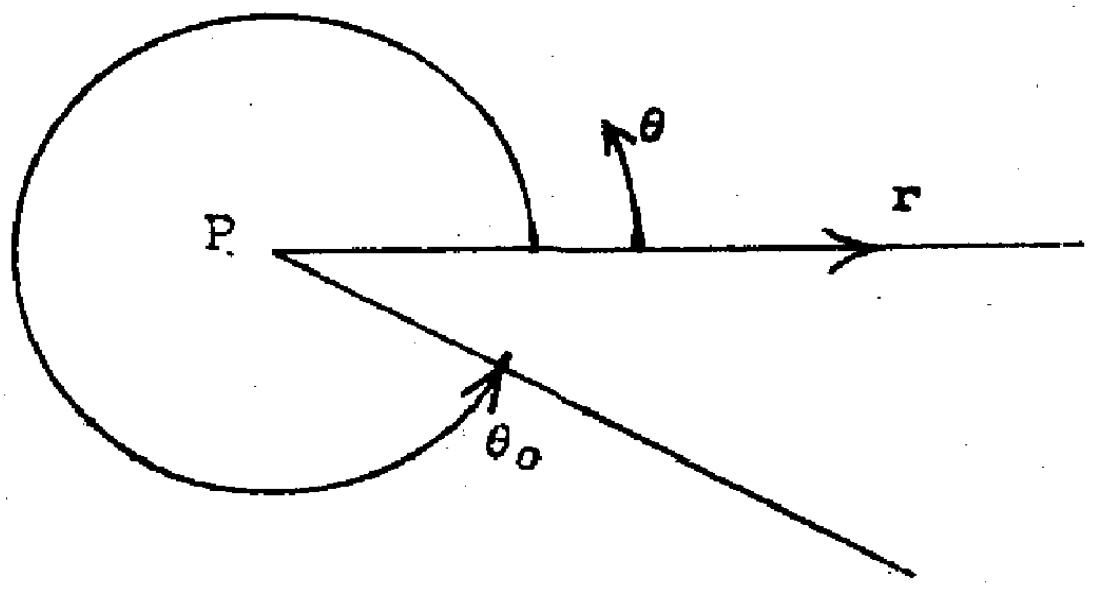

Fig. 1 .

Typical Corner.

where $w(r, \theta)$ is a solution of Laplace's equation and represents the steady-state form of the singularity, we obtain the following equations for $\mathrm{R}$ and $\psi$

$$
\begin{aligned}
& \psi^{\prime \prime}=-\omega^{2} \psi, \\
& \mathrm{R}^{\prime \prime}+\frac{1}{\mathrm{r}} \mathrm{R}^{\prime}+\mathrm{R}\left(\alpha^{2}-\frac{\omega^{2}}{\mathrm{r}^{2}}\right)=0 .
\end{aligned}
$$

Equation (2.7) has a solution

$$
\psi=\mathrm{A} \cos \omega \theta+\mathrm{B} \sin \omega \theta
$$

and (2.8) is Bessel's equation with solution

$$
\mathrm{R}=\mathrm{CJ} \omega(\alpha \mathrm{r}) \quad, \mathrm{w} \geq 0,
$$

which is finite at $\mathrm{P}$.

Thus, a separation of variables solution of (2.5) is

$$
\mathrm{u}(\mathrm{r}, \theta, \mathrm{t})=\mathrm{e}^{\alpha^{2} \mathrm{t}} \mathrm{J} \omega(\alpha \mathrm{r})[\mathrm{A} \cos \omega \theta+\mathrm{B} \sin \omega \theta]+\mathrm{w}(\mathrm{r}, \theta) .
$$


Imposing the boundary conditions (2.6) on the above solution we obtain

$$
\begin{aligned}
& \mathrm{B}=\mathrm{0}, \\
& \omega=\mathrm{k} \pi / \theta_{\mathrm{o}}, \quad \mathrm{k}=0,1,2, \ldots .
\end{aligned}
$$

where (Motz 1946)

$$
\mathrm{w}(\mathrm{r}, \theta)=\sum_{\mathrm{k}=0}^{\infty} \mathrm{c}_{\mathrm{k}} \mathrm{r}^{\mathrm{k}^{\lambda}} \quad \cos \mathrm{k} \lambda \theta
$$

with $\lambda=\pi / \theta_{\mathrm{o}}$ a solution of (2.5) and (2.6) becomes

$$
\mathrm{u}(\mathrm{r}, \theta, \mathrm{t})=\sum_{\mathrm{K}=0}^{\infty} \mathrm{A}_{\mathrm{k}} \mathrm{e}^{-\alpha^{2}} \mathrm{~J}_{\mathrm{k}_{\lambda}}(\alpha \mathrm{r}) \cos \mathrm{k} \lambda \theta+\sum_{\mathrm{K}=0}^{\infty} \mathrm{c}_{\mathrm{k}} \mathrm{r}^{\mathrm{k}^{\lambda}} \cos \mathrm{k} \lambda \theta
$$

A general solution in the neighbourhood of $\mathrm{P}$ will be

$$
\mathrm{u}=\sum_{\mathrm{j}=0}^{\infty} \sum_{\mathrm{K}=0}^{\infty} \mathrm{A}_{\mathrm{k}} \mathrm{j} \mathrm{e}^{-\alpha \mathrm{j}^{2 \mathrm{t}}} \mathrm{j}_{\mathrm{k} \lambda}\left(\alpha_{\mathrm{j}} \mathrm{r}\right) \cos \mathrm{k} \lambda \theta+\sum_{\mathrm{K}=0}^{\infty} \mathrm{c}_{\mathrm{k}} \mathrm{r}^{\mathrm{k} \lambda} \cos \mathrm{k} \lambda \theta
$$

The $\alpha_{j}$ 's are the zeros of some function of Beasel functions determined by the conditions on another part of the boundary.

The solution, (2.11), at point 0 is finite but successive derivatives with respect to $\mathrm{r}$ at $\mathrm{P}$ may not be finite. In fact, If $\theta_{0}>\pi(\lambda<1)$ then all derivatives of u with respect to $r$ will contain singular terms. This indicates the necessity for replacing the finite-difference approximation at $\mathrm{P}$.

3. Numerical Treatment of Singularities.

The method to be described is essentially that of Motz (1946) and is applied to the problem of the re-entrant corner, defined by 


$$
\theta_{\mathrm{o}}=\frac{3 \pi}{2} \text { i. e. } \quad \lambda=\frac{2}{3}
$$

Therefore equation (2.11) becomes

$$
\mathrm{u}(\mathrm{r}, \theta, \mathrm{t})=\sum_{\mathrm{j}=0}^{\infty} \sum_{\mathrm{K}=0}^{\infty} \mathrm{A}_{\mathrm{k}} \mathrm{j} \mathrm{e}^{-\alpha \mathrm{j}^{2} \mathrm{t}} \frac{\mathrm{J}_{2 \mathrm{k}}}{3}(\alpha \mathrm{jr}) \cos \frac{2 \mathrm{k} \theta}{3}+\sum_{\mathrm{K}=0}^{\infty} \mathrm{c}_{\mathrm{k}} \mathrm{r}^{\frac{2 \mathrm{k}}{\mathrm{a}}} \cos \frac{2 \mathrm{k} \theta}{3}
$$

Since we are interested in approximating to the solution in the neighbourhood of $\mathrm{P}$ we express equation (3.1) as a single power series in $r$ by expanding the Bessel functions and collecting terms of like powers in $r$. Thus

$$
\frac{\mathrm{J}_{2 \mathrm{k}}(\mathrm{z})}{3}=\sum_{\mathrm{m}=\mathrm{o}}^{\infty} \frac{(-1)^{\mathrm{m}\left(\frac{\mathrm{z}}{2}\right)^{\frac{2 \mathrm{k}}{3}+2 \mathrm{~m}}}}{\mathrm{~m} ! \mathrm{r}\left(\frac{2 \mathrm{k}}{3}+\mathrm{m}+1\right)}
$$

and (3-1) can be written

$$
\begin{aligned}
\mathrm{u}= & \mathrm{a}_{\mathrm{o}}(\mathrm{t})+\mathrm{a}_{1}(\mathrm{t}) \cos \frac{2 \theta}{3} \mathrm{r}^{2 / 3}+\mathrm{a}_{2}(\mathrm{t}) \cos \frac{4 \theta}{3} \mathrm{r}^{4 / 3} \\
& +\mathrm{r}^{2}\left[\mathrm{a}_{3}(\mathrm{t}) \cos 2 \theta-b_{\circ}(t)\right]+0\left(r^{8 / 3}\right),
\end{aligned}
$$

where the a's are functions of $t$ and represent the coefficients of the leading term in each Bessel function expansion. The term $a_{0}$ is the coefficient of the first term of $J_{o}$ and is given by

$$
a_{o}(t)=c_{o}+\sum_{j=0}^{\infty} A_{o} j e^{-\alpha j^{2} t}
$$


Similarly $a_{1}$ corresponds to the leading term of the expansion of $J_{1}$ and etc. The b's are the coefficients of the second terra in each Bessel function expansion and so on.

The series (3.2) is used, at time t, to obtain function values at points near to the corner in terms of those at points further away. The two sets of points will be referred to as Near and Far points. The number of terms considered in the series determines the number of Near and Far points used as one Hear and one Far point are required to eliminate each unknown coefficient. Inspection of (3.2) reveals that for only three terms to be taken account of, three Near and three Far points are required, whereas, if four terms are used five pairs of points are needed.

In the case of just three terms "being used in the approximation, we have

$$
u=a_{o}+a_{1} \cos \frac{2 \theta}{3} r^{2 / 3}+a_{2} \cos \frac{4 \theta}{3} r^{4 / 3}
$$

which can be rewritten as

$$
\mathrm{u}=\left[1, \cos \frac{2 \theta}{3} \mathrm{r}^{2 / 3}, \cos \frac{4 \theta}{3} \mathrm{r}^{4 / 3}\right]\left[\mathrm{a}_{\mathrm{o}}, \mathrm{a}_{1}, \mathrm{a}_{2}\right]:
$$

In figure $2 \mathrm{~N}_{1}, \mathrm{~N}_{2}, \mathrm{~N}_{3}$ are the $\mathrm{Near}$ points and $\mathrm{F}_{1}, \mathrm{~F}_{2}, \mathrm{~F}_{3}$ are the Far points. 


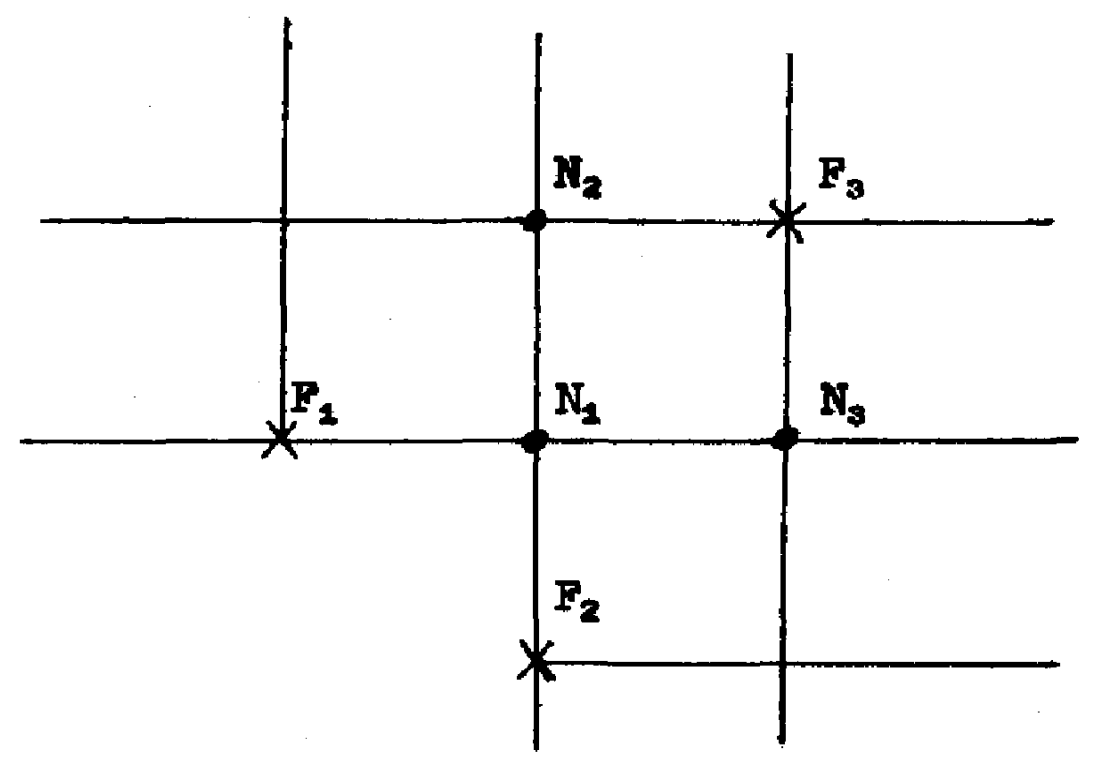

Fig.2. Near and Far Points

From (3.3) we can obtain the relationships:-

$$
\underline{\mathrm{U}}_{\mathrm{N}}=\mathrm{N}_{\underline{\mathrm{a}}} \quad, \quad \underline{\mathrm{u}}_{\mathrm{F}}=\mathrm{F}_{\mathrm{a}}
$$

where $\underline{u}_{\mathrm{N}}$ and $\underline{\mathrm{u}}_{\mathrm{F}}$ are vectors of the function values at Near and Far points respectively, and

$$
\underline{\mathrm{a}}=\left[\mathrm{a}_{0}, \mathrm{a}_{1}, \mathrm{a}_{2}\right]^{\prime} .
$$

Eliminating a from the equations (3.4) gives

$$
\underline{\mathrm{u}}_{\mathrm{N}}=\mathrm{NF}^{-1} \underline{\mathrm{u}}_{\mathrm{F}}
$$

and this relationship is used whenever function values at $\mathrm{N}_{1}, \mathrm{~N}_{2}$ or $\mathrm{N}_{3}$ are required by the explicit finite-difference process. Since this scheme uses only function values at time t to compute values at $\mathrm{t}+\delta \mathrm{t}$, the application of (3.5) at $t$, when required, will in no way alter the explicit nature of the scheme. 
Any number of terms can be used in the approximation. The more terms considered, the better the analytical representation of the singularity, but a. higher order approximation does not necessarily imply greater numerical accuracy, since the gain from a better approximation is offset by the enlarged region needed to represent it. Ideally, to improve accuracy we need to increase the order of the approximation without substantially increasing the region of application, that is, try to restrict Near and Far points to the neighbourhood of P.

One way of achieving this is to introduce a finer mesh just around the re-entrant corner, but the method becomes cumbersome when programmed. Another, is to choose some Near points at non-tabular points and then ignore them when applying the difference scheme.

For example, consider an approximation containing six terms, by further inspection of (3.2) it is found that nine Near and Par points are required. Arranging them as shown in figure 3 gives greater accuracy for the same number of mesh points as are required for the normal application of a five term approximation. 


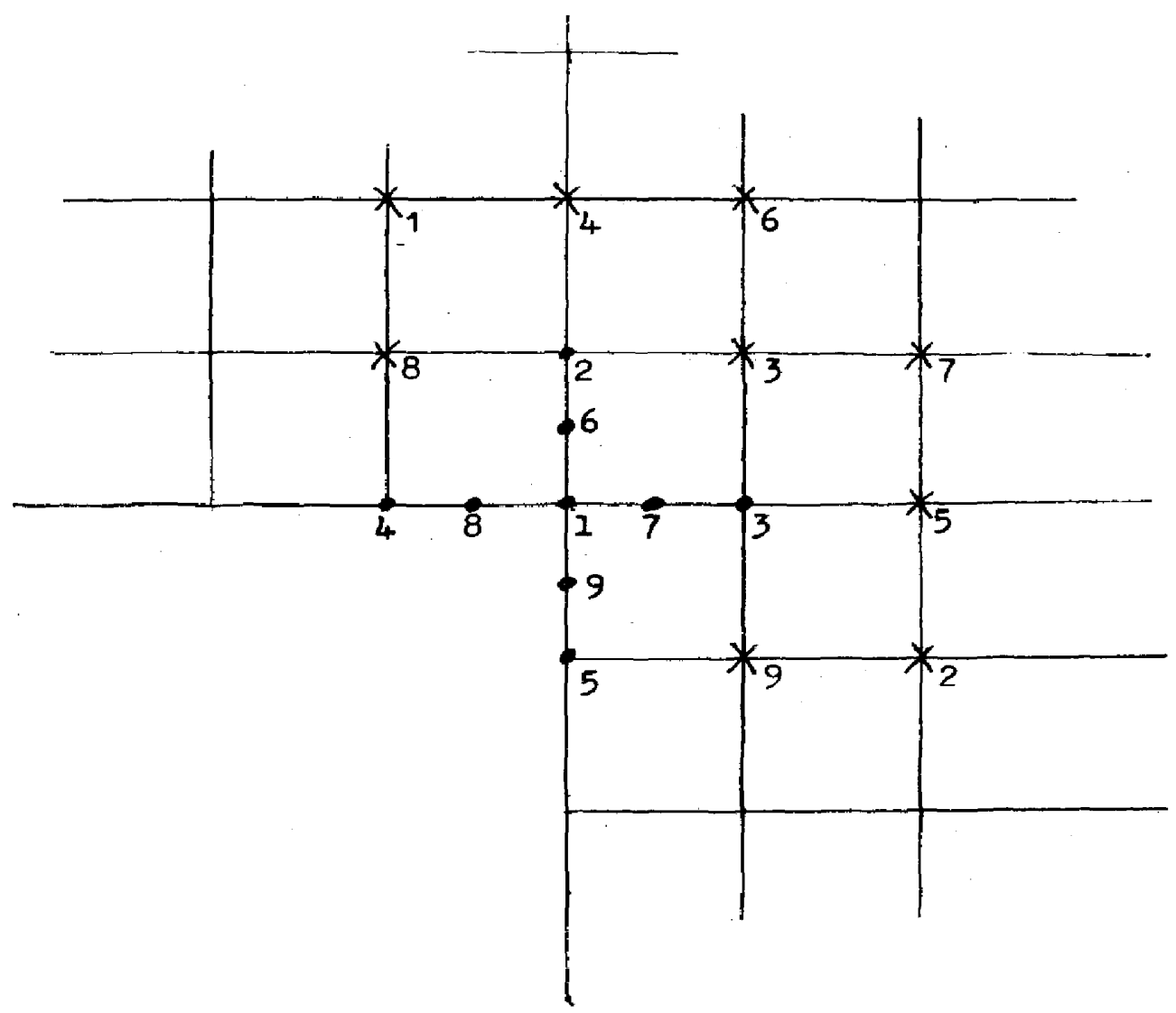

Far points denoted by $x$, Near points denoted by.

(Total number of mesh points used is 14 as opposed to 18)

fig. 3

The Near points numbered $6,7,8$ and 9 in figure 3 are used solely for the elimination of coefficients in the approximation and play no other part in the step by step finite-difference process. 
11.

4. Model Problems.

4.1. First Problem.

Consider the problem, $u(x, y, t)$ satisfying the

equation

$$
\mathrm{v}^{2} \mathrm{u}=\frac{\partial \mathrm{u}}{\partial \mathrm{t}}, \quad \mathrm{t}>0
$$

in the region $\mathrm{R}$, shown in figure 4, with the boundary conditions

$$
\begin{aligned}
& \mathrm{u}(\mathrm{o}, \mathrm{y}, \mathrm{t})=1000, \\
& \mathrm{u}(1, \mathrm{y}, \mathrm{t})=0,
\end{aligned}
$$

and $\frac{\partial \mathrm{u}}{\partial \mathrm{v}}=0$ on all other boundaries, where $\frac{\partial}{\partial \mathrm{v}}$ is the derivative normal to the boundary. The initial condition is a small time solution in a plane medium and is taken to be

$$
\mathrm{u}(\mathrm{x}, \mathrm{y}, 0)=\operatorname{erfc}\left(\frac{\mathrm{x}}{2 \sqrt{\mathrm{t}}}\right)
$$

where $\mathrm{t}=0.0005$ and is equivalent to one time step in the numerical solution which follows.

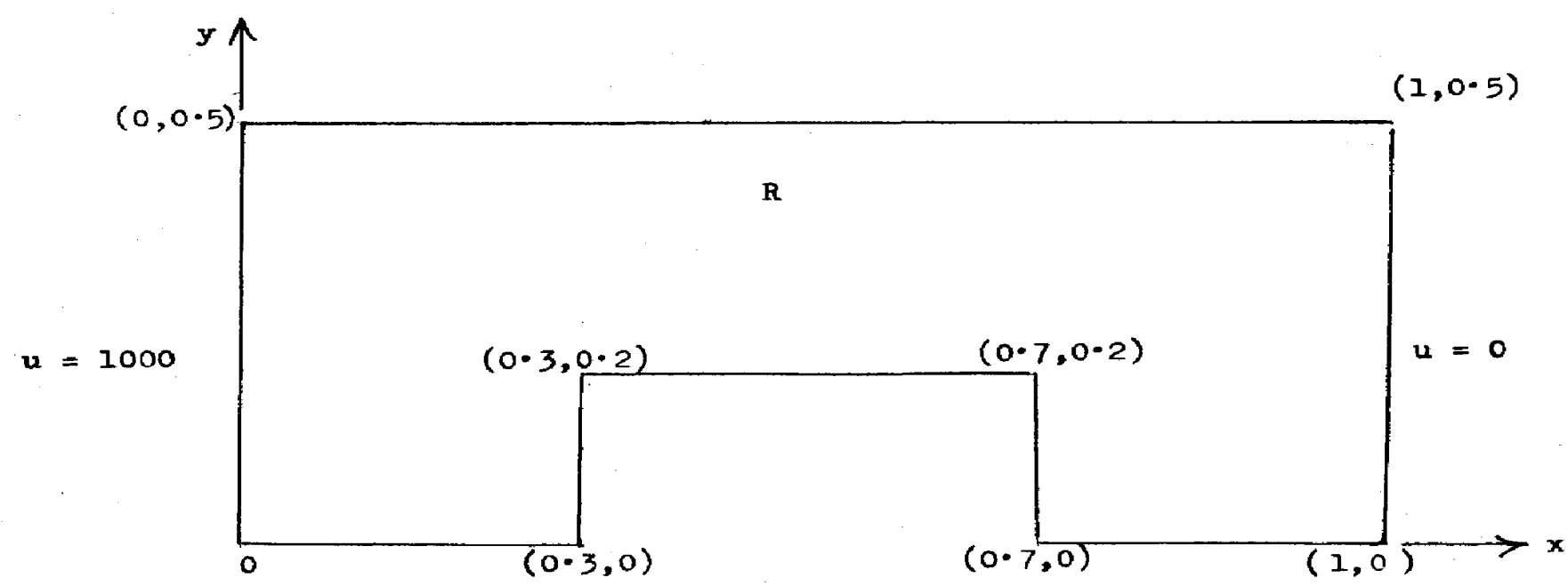

Fig 4. solution Domain 
$\underline{\text { TABLE } 1}$

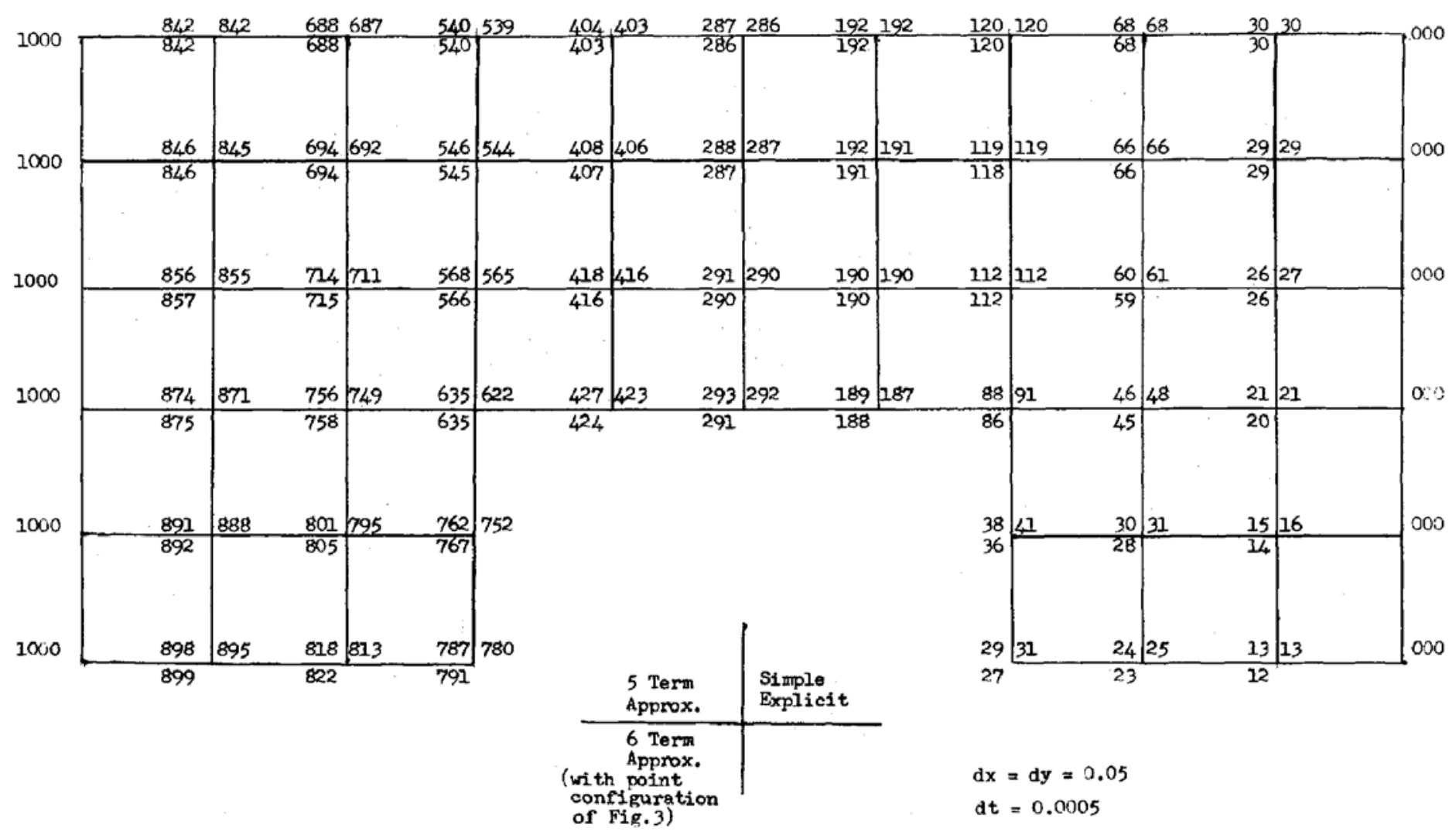

Solution corresponding to a time of 0.1 ( 200 time steps) 
The problem was solved with three, five and six term approximations at the two singular points. A mesh length of 0.05 was used, and the results presented in Table 1 are after 200 time steps of 0.0005 . For comparison the results obtained from the standard application of the explicit finite-difference scheme with no special regard for the corner singularities are also shown.

Results obtained using a three term approximation are not tabulated as they proved to be totally inadequate and it would appear that on such a grid three terms are not sufficient to represent the solution around each singularity.

\subsection{Second Problem.}

Consider the first problem again, but in the region illustrated in figure 5. Here, the singularities are closer together and this severely restricts the number of terms that can be used in the approximation. It was found, using the same mesh as in the first problem, that any approximation with more than five terms failed to give an acceptable solution, except for the special case of the six term approximation with the point arrangement of figure 3. To use a higher order approximation a smaller mesh is required which increases computation time considerably.

Table 2 shows the results obtained using a six term approximation, firstly, applied in the usual way but with a finer mesh, and secondly, using the point configuration of figure 3 on the same mesh as in the previous problem. 
$\underline{\text { TABEL } 2}$

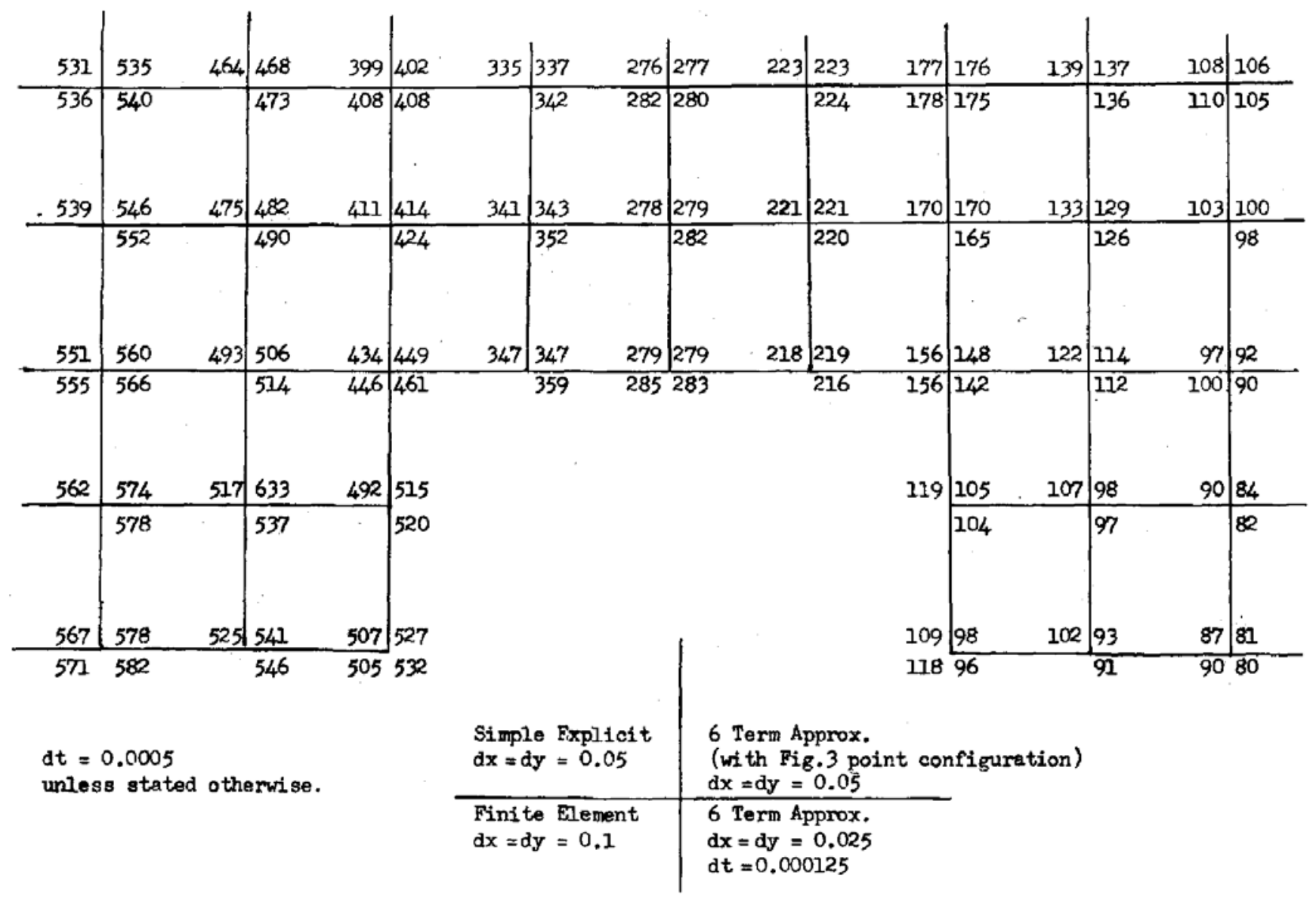

Solution corresponding to a time of $0 \cdot 1$ 


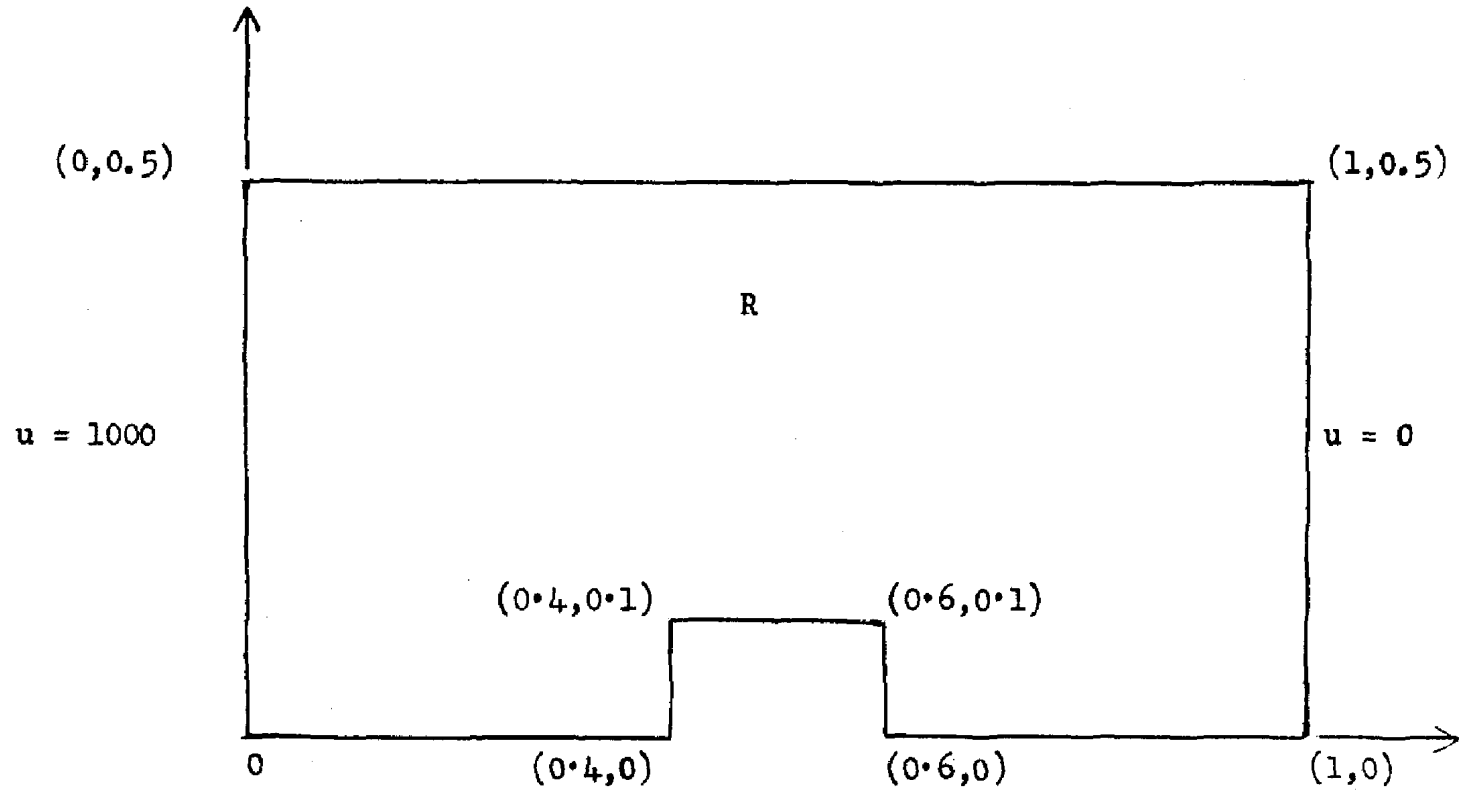

Fig.5.

Also tabulated are results obtained using the standard explicit method and the finite element scheme of Wilson and Nickell (1966). In this scheme triangular elements of side 0.1 were used with linear trial functions.

Table 2 only shows results around the two corners as values further away were consistent with each other.

\subsection{Discussion of Results.}

The results of both problems clearly illustrate the inadequacies of standard numerical methods in obtaining accurate approximations in the neighbourhood of boundary singularities, although the explicit method is surprisingly good especially in the first problem. This is because the five point finite-difference replacement inadvertently 
takes account of the first singular term in the exact solution for the re-entrant corner.

For example, consider the first two typical terms of equation (3.1). We have the form

$$
\begin{aligned}
\mathrm{u}(\mathrm{r}, \theta, \mathrm{t})= & \mathrm{A}_{\mathrm{oj}} \mathrm{e}^{-\alpha \mathrm{j}^{2} \mathrm{t}} \mathrm{J}_{\mathrm{o}}\left(\alpha_{\mathrm{j}} \mathrm{r}\right)+\mathrm{A}_{1 \mathrm{j}} \mathrm{e}^{-\alpha \mathrm{j}^{2} \mathrm{t}} \mathrm{J}_{2 / 3}\left(\alpha_{\mathrm{j}} \mathrm{r}\right) \cos \frac{2 \theta}{3} \\
& +\mathrm{c}_{\mathrm{o}}+\mathrm{c}_{1} \mathrm{r}^{2 / 3} \cos \frac{2 \theta}{3}
\end{aligned}
$$

corresponding to k being equal to zero and one in (3.1). We can now write down the form of the function values in the five points replacement, letting

$$
\mathrm{T}_{\mathrm{i}}=\mathrm{A}_{\mathrm{i} j} \mathrm{e}^{-\alpha \mathrm{j}^{2} \mathrm{t}} \mathrm{J}_{\frac{2 \mathrm{i}}{3}}(\alpha \mathrm{jh}), \quad \text { for } \mathrm{i}=0,1,
$$

we have,

$$
\begin{aligned}
& \mathrm{u}(\mathrm{h}, \mathrm{o}, \mathrm{t})=\mathrm{T}_{0}+\mathrm{T}_{1}+\mathrm{c}_{0}+\mathrm{c}_{1} \mathrm{~h}^{2 / 3}, \\
& \mathrm{u}\left(\mathrm{h}, \frac{\pi}{2}, \mathrm{t}\right)=\mathrm{T}_{\mathrm{o}}+\frac{1}{2} \mathrm{~T}_{1}+\mathrm{c}_{\mathrm{o}}+\frac{1}{2} \mathrm{c}_{1} \mathrm{~h}^{2 / 3}, \\
& \mathrm{u}(\mathrm{h} . \mathrm{w}, \mathrm{t})=\mathrm{T}_{\mathrm{o}}-\frac{1}{2} \mathrm{~T}_{1}+\mathrm{c}_{\mathrm{o}}-\frac{1}{2} \mathrm{c}_{1} \mathrm{~h}^{2 / 3}, \\
& \mathrm{u}\left(\mathrm{h}, \frac{3 \pi}{2}, \mathrm{t}\right)=\mathrm{T}_{\mathrm{o}}-\mathrm{T}_{1}+\mathrm{c}_{\mathrm{o}}-\mathrm{c}_{1} \mathrm{~h}^{2 / 3},
\end{aligned}
$$

and

$$
\mathrm{u}(\mathrm{o}, \theta, \mathrm{t})=\mathrm{T}_{\mathrm{o}}+\mathrm{c}_{0}, \quad \text { with } \mathrm{h}=0 \text { in } \mathrm{T}_{\mathrm{o}} \text {. }
$$

Therefore, on substituting for $\mathrm{T}_{0}$, the five point finitedifference replacement becomes 
$4 \mathrm{~A}_{\mathrm{o} j} \mathrm{e}^{-\alpha \mathrm{j}^{2} \mathrm{t}}\left[\frac{\mathrm{J}_{\mathrm{o}}(\alpha \mathrm{jh})-\mathrm{J}_{\mathrm{o}}(\mathrm{o})}{\mathrm{h}^{2}}\right]$,

which can be written, after expanding the Bessel functions,

as

$$
\begin{aligned}
& 4 \mathrm{~A}_{\mathrm{oj}} \mathrm{e}^{-\alpha \mathrm{j}^{2} \mathrm{t}}\left[\frac{\left(1-\left(\alpha_{\mathrm{j}_{2}} \mathrm{~h}\right)^{2}+\mathrm{O}\left(\mathrm{h}^{4}\right)\right)-(1)}{\mathrm{h}^{2}}\right], \\
& \text { i.e. } \\
& \quad-\mathrm{A}_{\mathrm{oj}}\left(\alpha_{\mathrm{j}}\right)^{2} \mathrm{e}^{-\alpha \mathrm{j}^{2} \mathrm{t}}+\mathrm{O}\left(\mathrm{h}^{2}\right),
\end{aligned}
$$

and as h becomes small, this expression approaches $\frac{\partial u}{\partial t}$ given by (3.1) at $r=0$. Therefore, the explicit finite-difference process satisfies the two leading terms of the exact solution.

The effect of treating the singularities is shown in the first problem and the results emphasize the gain in representation from the addition of an extraterm for the same number of Near and Far mesh points.

The second problem not only contain two singularities but the distance between them is relatively small. Although it is desirable to include as many terms as possible in an approximation the close proximity of the corners restricts the number of neighbouring mesh points available. Table 2 shows the results obtained from the application of two six term approximations. Naturally, the values obtained from the normal application on a finer mesh $(\mathrm{dx}=0.025, \mathrm{dt}=0.000125)$ 
are more accurate but the computation time is more than tenfold that of the special six term scheme on the usual $\operatorname{mesh}(\mathrm{dx}=0.05, \quad \mathrm{dt}=0.0005)$.

\section{Conclusions}

The results presented indicate the need to treat boundary singularities in Parabolic partial differential equations, if an accurate evaluation of function values in such regions is needed. The method described is one way of tackling this problem.

If, on the other hand, accurate values around a singularity are not required then the simple explicit method is probably adequate, as the results of both problems suggest that inaccuracies due to sharp corners are not propagated throughout the solution domain.

The author is indebted to Professor J. Crank for his guidance during the preparation of this paper. 


\section{REFERENCES}

1 Motz H. (1946). Quarterly Journal of Applied Mathematics. 4, 371-377.

2 .Wilson, E.L. \& Nickell R.E.(1966.) Nuclear Engineering and Design. 4, 276-286. 